\title{
IDENTIFICACIÓN DE AMBIENTES REPRESENTATIVOS Y DISCRIMINATORIOS PARA SELECCIONAR GENOTIPOS DE ARROZ MEDIANTE EL BIPLOT GGE ${ }^{1}$
}

\author{
Ismael Camargo-Buitrago ${ }^{2}$,Evelyn Quirós-Mc Intire ${ }^{2}$, Román Gordón-Mendoza ${ }^{2}$
}

\begin{abstract}
RESUMEN
Identificación de ambientes representativos y discriminatorios para seleccionar genotipos de arroz mediante el Biplot GGE. El objetivo del presente estudio fue identificar ambientes representativos y discriminatorios para seleccionar genotipos de arroz mediante el Biplot GGE. Se recurrió a la base de datos del proyecto de arroz periodo 2001-2009. Se analizaron: rendimiento de grano (toneladas/hectárea) y la proporción de granos enteros, de manera individual, y mediante un índice de selección (rendimiento + granos enteros). La información fue analizada mediante el programa Biplot GGE. A cada Biplot generado se le determinó la distancia en milímetros entre localidades verdaderas y la ideal; posteriormente las distancias fueron estandarizadas. Además se estimó la capacidad discriminatoria y representatividad de las localidades. A excepción de Alanje, las localidades más apropiadas para rendimiento (Soná, Barú), no fueron las mismas para granos enteros (Tonosí, Barú, Divisa). El índice de selección identificó las localidades apropiadas para seleccionar (Tonosí, Alanje, Calabacito, Soná, Barú). Todas las localidades fueron efectivas en su capacidad discriminatoria para rendimiento. Se encontraron diferencias en representatividad, siendo Calabacito y Changuinola, las de mayor y menor representatividad, respectivamente. Las localidades presentaron similar capacidad discriminatoria y representatividad para granos enteros. Al integrar rendimiento más granos enteros, se hizo posible separar las localidades más discriminatorias (Remedios, Tanara, Alanje) y las más representativas (Calabacito, Tonosí, Barú). Las implicaciones prácticas de este trabajo es que nos permitirá priorizar la investigación en aquellas localidades más apropiadas para identificar genotipos superiores.
\end{abstract}

Palabras claves: Selección arroz, interacción genotipoambiente, ambientes discriminatorios y representativos, ambiente ideal.

\begin{abstract}
Identification of representative and discriminatory environments to select genotypes using GGE Biplot. The objective of this work was to identify representative and discriminatory environments to select rice genotypes using the Biplot GGE technique. The rice data base of the Rice Project 2001-2009 was used. Garin yield and proportion of whole grains, individually and by the use of a selection index (yield + whole grains) were analyzed using Biplot GGE. Every Biplot generated was analyzed for distance in $\mathrm{mm}$. between real localities and the ideal; distances were later standardized. In addition, the discriminatory capacity and representativity of each locality were determined. With the exception of Alanje, the localities more appropriate for higher yields (Soná, Barú), were not the same for the obtention of more whole grains (Tonosí, Barú, Divisa). The selection index identified appropriate locations for select (Tonosí, Alanje, Calabacito, Soná, Barú). All localities were effective in their discriminatory capacity for yield. Differences in representativity were observed, with Calabacito and Changuinola occupying the highest and lowest positions, respectively. All localities showed similar discriminatory capacity and representativity for whole grains. Integrating yield and more whole grains it became posible to separate more discriminatory (Remedios, Tanara, Alanje) and more representative (Calabacito, Tonosí, Barú) locations. The practical implication of this work is that it allows us to prioritize research in localities more appropriate for the identification of superior genotypes.
\end{abstract}

Key words: Rice selection, genotype x environment interaction, discriminatory and representative environments, ideal environment.

\footnotetext{
1 Recibido: 14 de junio, 2011. Aceptado: 3 de octubre, 2011. Proyecto de Investigación del Instituto de Investigación Agropecuaria de Panamá (IDIAP), financiado parcialmente por el Fondo Regional de Tecnología Agropecuaria (FONTAGRO).

2 Instituto de Investigación Agropecuaria de Panamá. Apartado postal 0819-05850, El Dorado, Panamá 6A, Panamá. icamargo@cwpanama. net; gordon.roman@gmail.com; evelynitzel26@gmail.com
} 


\section{INTRODUCCIÓN}

La presencia de la interacción genotipo por ambiente, se expresa como una respuesta inconsistente de algunos genotipos, cuando son evaluados en diferentes ambientes provocando la alteración del orden de los genotipos, cuando nos movemos de un ambiente a otro. Numerosos métodos basados en diferentes principios matemáticos-estadísticos, han sido descritos y utilizados para la evaluación de la interacción genotipo por ambiente, estos permiten determinar la estabilidad y adaptabilidad fenotípica de los cultivares cuando son evaluados en diferentes localidades y años. Entre los más conocidos se pueden citar: Finlay y Wilkinson (1963), Eberhart y Russell (1966), Baker (1988), Zobel et al. (1988), Lin y Binns (1988), Cruz et al. (1989), Kang (1993), Yan et al. (2000).

La caracterización ambiental juega un rol preponderante para definir estrategias de selección en los programas de mejoramiento genético de cultivos. Es importante para efectuar la evaluación de nuevos cultivares en diferentes condiciones para estimar apropiadamente el potencial genético productivo y la estabilidad fenotípica de las variedades; además de proveer una estrategia confiable para seleccionar los mejores genotipos disponibles, para las nuevas localidades (Crossa 1990, Lillemo et al. 2004, Rodríguez et al. 2005, Glaz y Kang 2008).

Para lograr una agrupación adecuada de los ambientes en base a la identificación de problemas comunes, se deben considerar las relaciones del rendimiento de grano y otras características cuantitativas; tanto, los factores abióticos (precipitación, altitud, tipo suelo etc.), como los bióticos (presión de plagas y enfermedades), los cuales pueden afectar la capacidad discriminatoria de las localidades (Blanche 2005, Blanche y Myers 2006).

$\mathrm{Al}$ recurrir al rendimiento de grano como única variable de respuesta para definir las condiciones ambientales, la aplicación de técnicas multivariadas al estudio de la interacción genotipo por ambiente ha mostrado ventajas, con el enfoque global y la interpretación complementada con elementos gráficos. Entre los más usadas están los métodos de agrupamiento (Fox y Rosielle 1982, Lin 1982, análisis de factores (Westcott 1987, Peterson y Pfeiffer 1989) y análisis AMMI (Zobel et al. 1988, Gauch y Zobel 1997, Atlin et al. 2000, Yan et al. 2000, Yan y Kang 2003,
Trethowan et al. 2003, Yang et al. 2005, Thomason y Phillips 2006).

La categorización o agrupamiento de las localidades en mega-ambientes a través del modelo Biplot GGE, fue propuesto por Yan et al. (2001); posteriormente Yan y Hunt (2002), Yan y Kang (2003); introducen y discuten el concepto de ambiente "ideal", definiéndolo como aquel con la mejor capacidad discriminatoria y representatividad entre el conjunto de localidades estudiadas.

El modelo Biplot GGE ubica gráficamente la posición de la localidad "ideal" que es teórica, altamente discriminatoria y es representativa, la misma está basada en los datos de la ubicación media obtenida del conjunto de datos provenientes de los genotipos y ambientes evaluados (Yan y Kang 2003). La distancia gráfica en dos dimensiones entre la localidad verdadera y la ideal es una indicación de la conveniencia de la localidad, con respecto a su capacidad para discriminar genotipos y su representatividad como ambiente adecuado para seleccionar diferentes características en determinados ambientes (Blanche y Myers 2006).

Yan et al. (2000), Yan y Kang (2003) plantearon utilizar el modelo Biplot GGE para examinar la interacción genotipo-ambiente, mediante la capacidad de discriminar y la representatividad de los ambientes de prueba como una medida conveniente para definir mega ambientes homogéneos. De acuerdo a este modelo, los ambientes con baja puntuación en el PCA 2 tienen más representatividad y alta puntuación en el PCA 1 equivale a mayor capacidad discriminatoria de los genotipos en términos del efecto genotípico principal (Yan et al. 2000, Yan y Rajcan 2002). Una detallada descripción del método biplot GGE es presentada por Yan et al. (2000), Yan y Hunt (2002) y Yan y Kang (2003).

Basados en la propuesta presentada por Yan y Kang (2003), Blanche (2005), Blanche y Myers (2006), Baxevanos et al. (2008), el presente estudio tuvo como objetivo identificar ambientes representativos y discriminatorios para seleccionar genotipos de arroz mediante el Biplot GGE.

\section{MATERIALES Y MÉTODOS}

El Instituto de Investigación Agropecuaria de Panamá (IDIAP), a través del proyecto "Desarrollo de 
germoplasma mejorado de arroz", realiza anualmente evaluaciones de cultivares de ciclo precoz e intermedio en juegos separados, las actividades se realizaron en diez localidades ubicadas en las zonas productoras de este grano en el país. Los ensayos fueron establecidos en un diseño experimental de bloques completos al azar. El tamaño de la unidad experimental y la toma de datos se efectúo de acuerdo a los estándares internacionales para este tipo de ensayos.

Para realizar este estudio se recurrió a la base de datos del proyecto correspondiente al periodo 2001 a 2009, el hecho de que el juego de genotipos de ciclo precoz e intermedio, no haya sido el mismo durante el periodo de estudio, es irrelevante (Blanche 2005, Blanche y Myers 2006, Baxevanos et al. 2008); debido a que la respuesta de los cultivares fueron utilizadas para estimar las distancias estandarizadas, la capacidad discriminatoria y representatividad de las localidades para las características rendimiento y calidad de grano.

Dentro del sistema nacional de evaluación de cultivares del IDIAP, los ensayos de rendimientos de los cultivares precoces e intermedios normalmente son conducidos en diez localidades bajo condiciones de secano, este sistema representa el $75 \%$ de la superficie sembrada con arroz en el país (MIDA 2010).
Las localidades de evaluación fueron: Alanje, Barú, Remedios, Changuinola, Soná, Divisa, Calabacito, Penonomé, Tonosí y Tanara. Las mismas presentan condiciones de suelos que varían, en cuanto a textura, el $\mathrm{pH}$ varía entre muy ácido $(4,3)$, ácido $(>5,0)$ y poco ácido $(6,0)$, mientras el contenido de materia orgánica es bajo a excepción de Alanje (Cuadro 1).

Los datos obtenidos de las diez localidades a través de nueve años de investigación fueron analizados para determinar cuáles de estas localidades de prueba son las más convenientes para mejorar o hacer más eficiente y efectivo el trabajo de selección de los nuevos cultivares de arroz, con el objetivo de priorizar las actividades e instalarlas en estas localidades, cuando haya limitantes económicas o por disponibilidad de semilla del material élite. Las variables agronómicas analizadas en este estudio mediante análisis de varianza y separación de medias mediante la prueba de Duncan $(\alpha<0,05)$, fueron el rendimiento de grano en toneladas por hectárea y la proporción de granos enteros, primero de manera individual, y posteriormente como componente de un índice de selección conjunta (rendimiento + granos enteros); a destacar que ambas características son altamente influenciadas por las condiciones ambientales.

Cuadro 1. Localidades de pruebas en el cultivo de arroz, ubicación geográfica y algunas características físicasquímicas de los suelos. Instituto de Investigación Agropecuaria de Panamá (IDIAP). 2001-2009.

\begin{tabular}{|c|c|c|c|c|c|c|}
\hline \multicolumn{2}{|c|}{ Localidades } & \multirow{2}{*}{$\begin{array}{l}\text { Latitud } \\
\text { (Norte) }\end{array}$} & \multirow{2}{*}{$\begin{array}{c}\text { Longitud } \\
\text { (Oeste) }\end{array}$} & \multirow[t]{2}{*}{ Tipoł suelo } & \multirow{2}{*}{$\begin{array}{l}\text { Materia }+ \\
\text { orgánica }\end{array}$} & \multirow[t]{2}{*}{$\mathrm{pH}+$} \\
\hline Nombre & Abreviatura & & & & & \\
\hline Alanje & $\mathrm{AL}$ & $08^{\circ} 23^{\prime} 43^{\prime \prime}$ & $082^{\circ} 33^{\prime} 34,2^{\prime \prime}$ & $\mathrm{FA}^{\dagger}$ & 9,2 & 5,7 \\
\hline Barú & BA & $08^{\circ} 27^{\prime} 04,4 ”$ & $082^{\circ} 51^{\prime} 22,5^{\prime \prime}$ & A & 1,1 & 4,6 \\
\hline Remedios & RE & $08^{\circ} 77^{\prime}$ & $081^{\circ} 49^{\prime} 67^{\prime \prime}$ & $\mathrm{F}$ & 5,76 & 5,2 \\
\hline Changuinola & $\mathrm{CH}$ & $07^{\circ} 47^{\prime} 56^{\prime \prime}$ & $081^{\circ} 15^{\prime} 78^{\prime \prime}$ & FL & 2,05 & 5,6 \\
\hline Soná & SO & $07^{\circ} 47^{\prime} 65^{\prime \prime}$ & $081^{\circ} 15^{\prime} 75$ & FARC & 3,15 & 5,2 \\
\hline Divisa & DI & $08^{\circ} 07^{\prime} 33^{\prime \prime}$ & $080^{\circ} 41^{\prime} 32^{\prime \prime}$ & FARA & 0,19 & 5,2 \\
\hline Calabacito & $\mathrm{CA}$ & $08^{\circ} 5^{\prime}$ & $081^{\circ} 5$ & ARC & Trazas & 4,3 \\
\hline Penonomé & $\mathrm{PE}$ & $08^{\circ} 27^{\prime} 17^{\prime \prime}$ & $080^{\circ} 21^{\prime} 24^{\prime \prime}$ & FA & 1,6 & 5,8 \\
\hline Tonosí & TO & $07^{\circ} 23^{\prime} 23^{\prime \prime}$ & $080^{\circ} 27^{\prime} 43^{\prime \prime}$ & FARA & 3,9 & 6,0 \\
\hline Tanara & $\mathrm{TA}$ & $09^{\circ} 07^{\prime} 44^{\prime \prime}$ & $079^{\circ} 13^{\prime} 46^{\prime \prime}$ & FARA & 2,2 & 5,5 \\
\hline
\end{tabular}

\$ Fuente: Laboratorio de suelos, Instituto de Investigación Agropecuaria de Panamá.

$\dagger \mathrm{FA}=$ franco arenoso; $\mathrm{A}=$ arenoso; $\mathrm{F}=$ franco; FARA = franco arcillo-arenoso; $\mathrm{ARC}=$ arcilloso; $\mathrm{FL}=$ franco limoso. 
Los datos fueron analizados mediante el programa informático Biplot GGE (Yan 2001-2009), el cual generó las gráficas Biplot. Posteriormente en cada uno de los 36 Biplot (nueve años de estudio x dos tipos de ciclo reproductivo $\mathrm{x}$ dos variables agronómicas), se determinó la distancia en milímetros entre cada localidad verdadera y la localidad ideal (Figura 1). A continuación las distancias fueron estandarizadas o normalizadas, estimándose entonces la distancia media estandarizada y la desviación estándar de todas las localidades en cada Biplot. La estandarización indica a qué distancia se encuentra el valor respecto a la media, reflejando la distancia en términos de desviación estándar (Blanche y Myers 2006, Baxevanos et al. 2008).

Para determinar el índice de selección combinado (rendimiento de grano + proporción de granos enteros), la distancia de cada gráfica entre el sitio verdadero y el ideal fue medido y se utilizó una ponderación arbitraria donde se asignó una ponderación de $60 \%$ para rendimiento de grano y de $40 \%$ para granos enteros, por cada Biplot y ciclo de madurez (precoz e intermedio).

Las distancias estandarizadas entre las localidades verdaderas y la ideal, de cada uno de los nueve años de evaluación de los cultivares de ciclos precoces e intermedios, fueron utilizados para realizar un análisis de varianza, considerando las fuentes de variación años, localidades y la interacción año por localidad. Para la separación de las medias estandarizadas se utilizó la prueba de comparaciones múltiples de Duncan $(\alpha<0,05)$ y se estimó también, la desviación estándar para rendimiento de grano, proporción de granos enteros y el índice se selección (rendimiento + granos enteros).

La base de datos también fue utilizada para evaluar la capacidad discriminatoria y la representatividad de las localidades de prueba (Yan y Kang 2003, Baxevanos et al. 2008). La longitud del vector de las localidades es una medida de su capacidad de discriminar. A mayor longitud del vector más alta es la capacidad de discriminar. Por el contrario, localidades

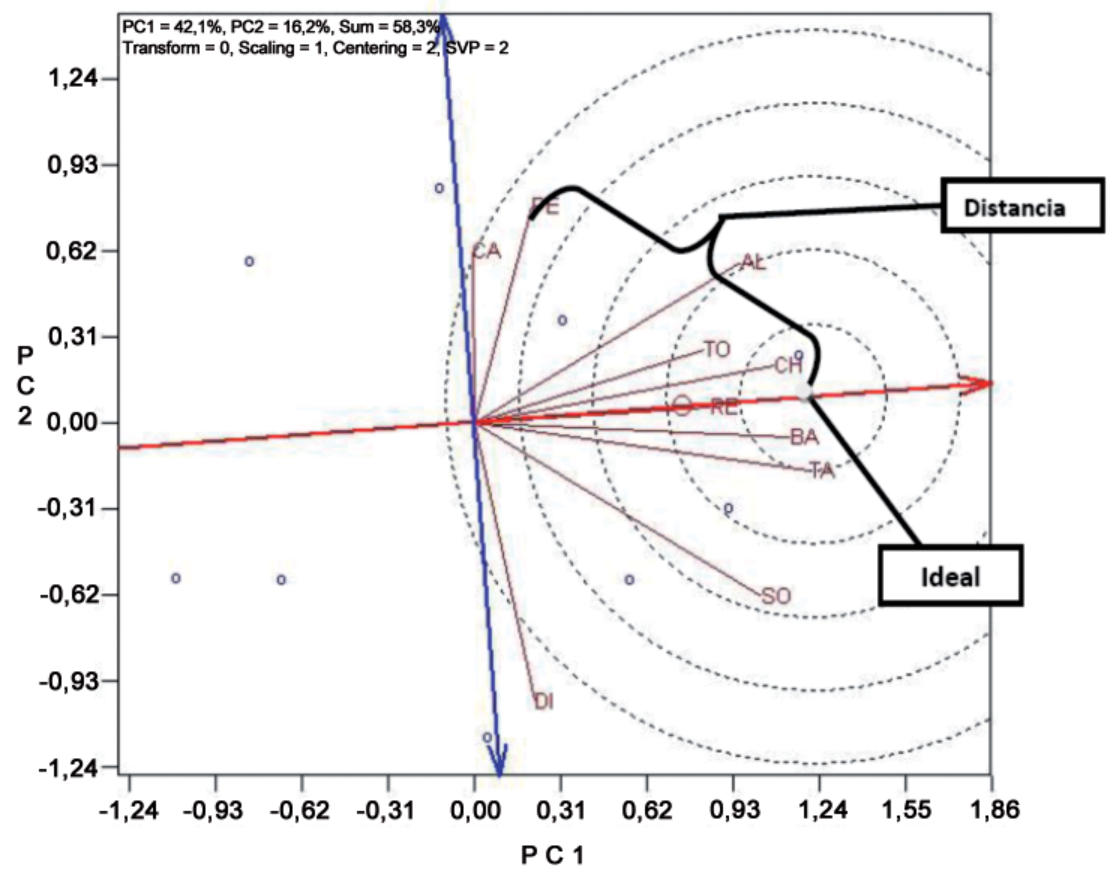

Figura 1. Clasificación de las localidades de prueba basados en la capacidad discriminatoria y representatividad del ambiente ideal (Biplot GGE) en arroz durante nueve años de estudio, dos ciclos reproductivos y dos variables agronómicas. Instituto de Investigación Agropecuaria de Panamá (IDIAP). 2001-2009. 
con proyecciones lejanas de la línea de estabilidad son considerados menos representativas. Para cada localidad evaluada, se estimó la capacidad discriminatoria y representatividad y sus respectivas desviaciones estándar para rendimiento de grano y proporción de granos enteros, de manera individual y considerando de manera integral ambas características (Figura 2).

\section{RESULTADOS Y DISCUSIÓN}

El análisis de varianza (Cuadro 2) aplicado a las distancias estandarizadas, de cada uno de los nueve años de evaluación, de los cultivares de ciclos precoces e intermedios, indica que para las variables rendimiento de grano, proporción de granos enteros y el

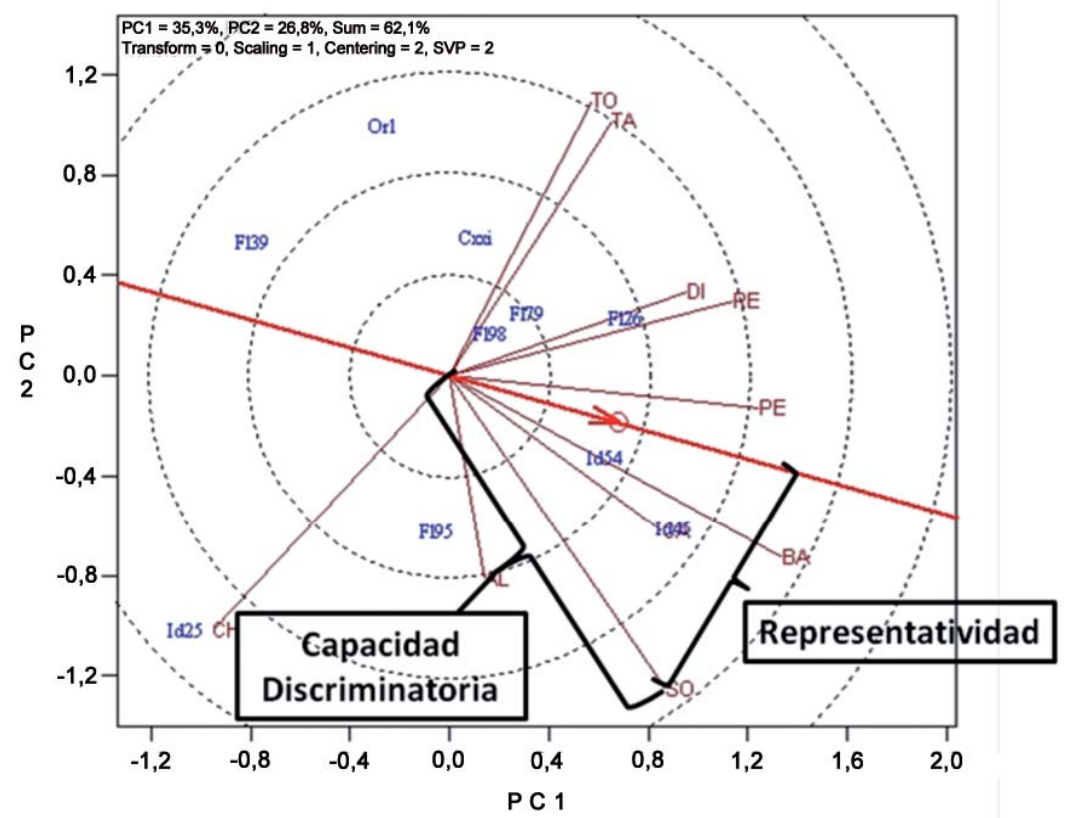

Figura 2. Determinación de la capacidad de discriminatoria y representatividad de las localidades de prueba para las características en estudio (Biplot GGE) en arroz durante nueve años de estudio, dos ciclos reproductivos y dos variables agronómicas. Instituto de Investigación Agropecuaria de Panamá (IDIAP). 2001-2009

Cuadro 2. Análisis de varianza de las distancias estandarizadas entre las localidades verdaderas y la ideal, para las variables rendimiento de grano, proporción de granos enteros y el índice de selección (rendimiento + granos enteros) en arroz. Instituto de Investigación Agropecuaria de Panamá (IDIAP). 2001-2009.

\begin{tabular}{lcccc}
\hline Fuente de variación & G.L. & Rendimiento de grano & Proporción de granos enteros & $\begin{array}{c}\text { Índice de selección } \\
\text { (rendimiento + granos enteros) }\end{array}$ \\
\cline { 3 - 5 } & & CME & CME & CME \\
\hline Años & 8 & $0,0538 N S$ & $0,0775 \mathrm{NS}$ & $0,1300 \mathrm{NS}$ \\
Localidades & 9 & $0,6298^{* *}$ & $0,7446^{* *}$ & $0,5378^{* *}$ \\
Años x localidades & 72 & $0,3519^{* *}$ & $0,3889^{* *}$ & $0,2289^{* *}$ \\
\hline
\end{tabular}

NS Indica que no existe diferencias significativas. ** Indica que existe diferencias altamente significativas $(\alpha<0,01)$. 
índice de selección (rendimiento + granos enteros), no hubo diferencias significativa para la fuente de variación años. Por el contrario, las tres variables mostraron diferencias altamente significativas para las fuentes de variación localidades y la interacción años x localidades. La no significancia de la fuente de variación años, reafirma que el efecto de genotipo en este estudio solamente es importante para generar la información para construir los Biplots (Blanche 2005, Blanche y Myers 2006, Baxevanos et al. 2008). Por otro lado, la significancia de localidades y de la interacción son las que permiten realizar las pruebas de comparación de las medias estandarizadas entre las localidades, para cada una de las variables.

Las distancias estandarizadas para rendimiento de grano entre las localidades verdaderas y la localidad ideal, la desviación estándar y clasificación de las diez localidades estudiadas (Cuadro 3), son presentadas en orden descendente; de acuerdo a su conveniencia para la selección de cultivares de arroz para rendimiento de grano.

Entre las localidades incluidas en este estudio, Soná, Alanje, Calabacito, Remedios, Tanara, Barú, Tonosí y Divisa tienen similar capacidad discriminatoria para seleccionar rendimiento de grano $(\alpha<0,05)$. Sin embargo, Penonomé y Changuinola resultaron las menos efectivas para estos propósitos, debido a su baja capacidad discriminatoria de los cultivares, o por no ser representativos de las localidades de prueba o de otras regiones productoras. Soná, Alanje y Calabacito, son localidades que se prestan para seleccionar para rendimiento de grano; Alanje, es la localidad de evaluación más antigua del proyecto de arroz y siempre ha sido considerado como un ambiente adecuado empíricamente para seleccionar germoplasma de arroz.

Por otro lado, Soná y Tonosí presentaron las desviaciones estándar más bajas entre las diez localidades, indicando una mayor aproximación al ambiente ideal; además, de presentar bajas fluctuaciones o variabilidad a través de los años (Blanche y Myers 2006, Baxevanos et al. 2008).

Las distancias estandarizadas clasificaron en orden decreciente las localidades más apropiadas para la selección de cultivares, para la variable calidad de granos específicamente la proporción de granos enteros (Cuadro 4). En este estudio, las localidades Soná, Alanje, Calabacito, Remedios, Tanara, Barú, Tonosí y Divisa resultaron estadísticamente similares en cuanto
Cuadro 3. Distancias estandarizadas y clasificación de las diez localidades estudiadas para seleccionar la característica rendimiento de grano en arroz. Instituto de Investigación Agropecuaria de Panamá (IDIAP). 2001-2009.

\begin{tabular}{lccc}
\hline Localidad & Clasificación & $\begin{array}{c}\text { Distancia } \\
\text { estandarizada }\end{array}$ & $\begin{array}{c}\text { Desviación } \\
\text { estándar }\end{array}$ \\
\hline Soná & 1 & $0,611 \mathrm{a}^{\dagger}$ & 0,29 \\
Alanje & 2 & $0,625 \mathrm{a}$ & 0,45 \\
Calabacito & 3 & $0,636 \mathrm{a}$ & 0,50 \\
Remedios & 4 & $0,684 \mathrm{ab}$ & 0,37 \\
Tanara & 5 & $0,741 \mathrm{ab}$ & 0,51 \\
Barú & 6 & $0,778 \mathrm{ab}$ & 0,55 \\
Tonosí & 7 & $0,828 \mathrm{ab}$ & 0,23 \\
Divisa & 8 & $0,912 \mathrm{ab}$ & 0,51 \\
Penonomé & 9 & $1,100 \mathrm{~b}$ & 0,57 \\
Changuinola & 10 & $1,114 \mathrm{~b}$ & 0,64 \\
\hline
\end{tabular}

Medias seguidas por la misma letra no difieren significativamente (Duncan $\alpha=0,05$ ).

a su eficiencia para seleccionar cultivares con mayor proporción de granos enteros $(\alpha<0,05)$. Al contrario, las localidades Penonomé y Changuinola, fueron las menos apropiadas para seleccionar esta característica. Tonosí, además de ser la localidad más apropiada para seleccionar calidad de grano, presenta la menor desviación estándar sugiriendo que esta localidad presenta la menor variabilidad entre las localidades estudiadas a través de los años, reflejando así, su proximidad a la localidad ideal.

Los resultados muestran que hay diferencias entre las localidades (Cuadros 3 y 4), en cuanto a su conveniencia para ser utilizados en la selección de características específicas, es decir que a excepción de Alanje, las localidades ventajosas a seleccionar para rendimiento de grano no fueron las mismas para seleccionar granos enteros, resultados similares obtuvieron Blanche y Myers (2006) y Baxevanos et al. (2008).

Normalmente cuando se seleccionan a través de localidades y años, no se hace la selección para una característica específica, salvo casos muy especiales de enfermedades (hotpots), sino que se incluye una serie de características determinantes para la aceptación 
Cuadro 4. Distancias estandarizadas y clasificación de las diez localidades estudiadas para seleccionar la característica proporción de granos enteros en arroz. Instituto de Investigación Agropecuaria de Panamá (IDIAP). 2001-2009.

\begin{tabular}{lccc}
\hline Localidad & Clasificación & $\begin{array}{c}\text { Distancia es- } \\
\text { tandarizada }\end{array}$ & $\begin{array}{c}\text { Desviación } \\
\text { estándar }\end{array}$ \\
\hline Tonosí & 1 & $0,356 \mathrm{a}^{\dagger}$ & 0,24 \\
Alanje & 2 & $0,636 \mathrm{ab}$ & 0,48 \\
Barú & 3 & $0,636 \mathrm{ab}$ & 0,45 \\
Divisa & 4 & $0,708 \mathrm{ab}$ & 0,58 \\
Soná & 5 & $0,743 \mathrm{abc}$ & 0,46 \\
Calabacito & 6 & $0,752 \mathrm{abc}$ & 0,52 \\
Tanara & 7 & $0,913 \mathrm{bc}$ & 0,55 \\
Remedios & 8 & $0,942 \mathrm{bc}$ & 0,49 \\
Penonomé & 9 & $0,983 \mathrm{bc}$ & 0,45 \\
Changuinola & 10 & $1,197 \mathrm{~b}$ & 0,61 \\
\hline
\end{tabular}

${ }^{\dagger}$ Medias seguidas por la misma letra no difieren significativamente (Duncan $\alpha=0,05$ ).

de las variedades por parte de los beneficiarios de la tecnología (productores, molineros y consumidores). Es necesario diseñar estrategias de selección para alcanzar las metas propuestas, una opción es utilizar índices para la selección de características múltiples; en este caso, se validó el índice de selección empírico propuesto por Blanche (2005), Blanche y Myers (2006), y validado por Baxevanos et al. (2008).

La distancia estandarizada y la clasificación de las localidades para la selección simultánea de las características rendimiento de grano y proporción de granos enteros (Cuadro 5), expresa la clasificación de las localidades identificadas como las más apropiadas para la selección de ambos caracteres; indicando que, Tonosí, Alanje, Calabacito, Soná y Barú podrían ser las localidades de evaluación más conveniente para seleccionar rendimiento de grano y proporción de granos enteros, simultáneamente $(\alpha<0,05)$. Además sitios como Tanara, Divisa y Remedios también pueden ser recomendados para hacer la selección simultánea.

Es conveniente resaltar que Penonomé y Changuinola fueron los ambientes menos apropiados para hacer trabajos de selección visualizando la liberación de nuevos cultivares de arroz con alto potencial de rendimiento y buena calidad de grano (Cuadro 5). Los resultados también destacan que es más conveniente estimar las distancias estandarizadas en función de un índice de selección o para seleccionar simultáneamente varias características, e identificar las localidades más convenientes en la selección de germoplasma de arroz, ya que se aproxima más al trabajo rutinario de selección que normalmente realizan los mejoradores.

En relación a estas dos localidades (Penonomé y Changuinola), los resultados fueron consistentes (Cuadros 3, 4 y 5) en el sentido de que no ofrecen ventajas para hacer mejoramiento genético; de manera que pueden ser excluidas de la red de localidades del sistema de evaluación con baja afectación para la toma de decisión. La respuesta en estas localidades puede deberse a que la selección no es significativa o simplemente no representan los otros sitios de prueba. Penonomé es la localidad donde se presenta la precipitación más baja del país, sometiendo el cultivo a constante estrés hídrico y a estrés biótico; por el contrario, Changuinola representa el sitio más lluvioso y es el único ubicado en la costa del Caribe panameño.

El estudio demostró que Alanje es una localidad apropiada para seleccionar individualmente rendimiento de grano y granos enteros, mediante la aplicación del índice de selección empírico (Blanche 2005, Blanche y Myers 2006). Esta localidad también es conveniente para seleccionar ambas características (Cuadro 5). Este estudio científico viene a confirmar la percepción empírica de que Alanje es el mejor ambiente de Panamá para seleccionar germoplasma de arroz entre las localidades evaluadas.

La segunda etapa del estudio consistió en estimar la capacidad discriminatoria y la representatividad de las diez localidades para seleccionar las características rendimiento de grano y proporción de granos enteros.

Las diez localidades fueron igualmente efectivas en cuanto a la capacidad discriminatoria, asociada al proceso de selección considerando exclusivamente rendimiento de grano. En cuanto a la representatividad, las localidades: Calabacito, Alanje, Remedios, Tanara, Soná, Barú, Tonosí, presentaron similar representatividad $(\alpha<0,05)$, para seleccionar la característica rendimiento de grano; no obstante, Changuinola, Divisa y Penonomé, fueron las menos representativas (Cuadro 6).

En relación a la característica proporción de granos enteros, las diez localidades evaluadas presentaron distancias estadísticamente similares $(\alpha<0,05)$ en su 
Cuadro 5. Distancias estandarizadas promedio y clasificación promedio de las diez localidades estudiadas para seleccionar simultáneamente para rendimiento de grano y proporción de granos enteros en arroz. Instituto de Investigación Agropecuaria de Panamá (IDIAP). 2001-2009.

\begin{tabular}{lccccc}
\hline Localidad & $\begin{array}{c}\text { Clasificación } \\
\text { Rendimiento } \\
\text { grano }\end{array}$ & $\begin{array}{c}\text { Clasificación } \\
\text { Granos } \\
\text { enteros }\end{array}$ & $\begin{array}{c}\text { Distancia * pro- } \\
\text { medio (Rend+ } \\
\text { GE) }\end{array}$ & $\begin{array}{c}\text { Desviación } \\
\text { estándar }\end{array}$ & $\begin{array}{c}\text { Clasificación } \\
\text { promedio }\end{array}$ \\
\hline Tonosí & 7 & 1 & $0,557 \mathrm{a}^{\dagger}$ & 0,25 & 1 \\
Alanje & 2 & 2 & $0,599 \mathrm{a}$ & 0,33 & 2 \\
Calabacito & 3 & 6 & $0,609 \mathrm{a}$ & 0,35 & 3 \\
Soná & 1 & 5 & $0,639 \mathrm{a}$ & 0,19 & 4 \\
Barú & 6 & 3 & $0,720 \mathrm{a}$ & 0,37 & 5 \\
Tanara & 5 & 7 & $0,758 \mathrm{ab}$ & 0,39 & 6 \\
Divisa & 8 & 4 & $0,804 \mathrm{ab}$ & 0,28 & 7 \\
Remedios & 4 & 8 & $0,836 \mathrm{ab}$ & 0,33 & 8 \\
Penonomé & 9 & 9 & $1,031 \mathrm{bc}$ & 0,41 & 9 \\
Changuinola & 10 & 10 & $1,129 \mathrm{c}$ & 0,51 & 10 \\
\hline
\end{tabular}

${ }^{\dagger}$ Medias seguidas por la misma letra no difieren significativamente (Duncan $\alpha=0,05$ ).

Cuadro 6. Distancias estandarizadas y desviaciones estándar para capacidad discriminatoria y representatividad, para la característica rendimiento de grano en arroz en las diez localidades evaluadas. Instituto de Investigación Agropecuaria de Panamá (IDIAP). 2001-2009.

\begin{tabular}{lcccccc}
\hline Localidades & $\begin{array}{c}\text { Clasificación } \\
\text { capacidad dis- } \\
\text { criminatoria }\end{array}$ & $\begin{array}{c}\text { Distancia para } \\
\text { capacidad dis- } \\
\text { criminatoria } \\
\text { rendimiento }\end{array}$ & $\begin{array}{c}\text { Desviación } \\
\text { estándar capa- } \\
\text { cidad } \\
\text { discriminatoria }\end{array}$ & $\begin{array}{c}\text { Clasificación re- } \\
\text { presentatividad }\end{array}$ & $\begin{array}{c}\text { Distancia para } \\
\text { representativi- } \\
\text { dad rendimiento }\end{array}$ & $\begin{array}{c}\text { Desviación } \\
\text { estándar repre- } \\
\text { sentatividad }\end{array}$ \\
\hline Tanara & 1 & $0,618 \mathrm{a}^{\dagger}$ & 0,40 & 4 & $0,686 \mathrm{ab}^{\dagger}$ & 0,44 \\
Remedios & 2 & $0,641 \mathrm{a}$ & 0,42 & 3 & $0,587 \mathrm{a}$ & 0,42 \\
Tonosí & 3 & $0,687 \mathrm{a}$ & 0,45 & 7 & $0,891 \mathrm{ab}$ & 0,62 \\
Soná & 4 & $0,724 \mathrm{a}$ & 0,52 & 5 & $0,742 \mathrm{ab}$ & 0,44 \\
Barú & 5 & $0,727 \mathrm{a}$ & 0,45 & 6 & $0,785 \mathrm{ab}$ & 0,42 \\
Calabacito & 6 & $0,742 \mathrm{a}$ & 0,54 & 1 & $0,491 \mathrm{a}$ & 0,65 \\
Divisa & 7 & $0,752 \mathrm{a}$ & 0,53 & 9 & $1,030 \mathrm{bc}$ & 0,53 \\
Alanje & 8 & $0,830 \mathrm{a}$ & 0,57 & 2 & $0,582 \mathrm{a}$ & 0,31 \\
Changuinola & 9 & $0,946 \mathrm{a}$ & 0,59 & 8 & $1,011 \mathrm{bc}$ & 0,64 \\
Penonomé & 10 & $1,057 \mathrm{a}$ & 0,64 & 10 & $1,059 \mathrm{c}$ & 0,52 \\
\hline
\end{tabular}

${ }^{\dagger}$ Medias seguidas por la misma letra no difieren significativamente $(\alpha=0,05)$.

capacidad discriminatoria y representatividad (Cuadro 7), indicando que no existen ventajas prácticas al seleccionar exclusivamente para esta característica específica.
Durante el proceso de selección, al realizar mejoramiento genético de cultivos, diferentes características son consideradas de manera integral, por ello, se estimó la capacidad discriminatoria y 
Cuadro 7. Distancias estandarizadas y desviaciones estándar para capacidad discriminatoria y representatividad, para la característica proporción de granos enteros de arroz en las diez localidades evaluadas. Instituto de Investigación Agropecuaria de Panamá (IDIAP). 2001-2009.

\begin{tabular}{lcccccc}
\hline Localidades & $\begin{array}{c}\text { Clasificación } \\
\text { capacidad } \\
\text { discriminatoria }\end{array}$ & $\begin{array}{c}\text { Distancia para } \\
\text { capacidad dis- } \\
\text { criminatoria } \\
\text { granos enteros }\end{array}$ & $\begin{array}{c}\text { Desviación es- } \\
\text { tándar } \\
\text { capacidad } \\
\text { discriminatoria }\end{array}$ & $\begin{array}{c}\text { Clasificación } \\
\text { representati- } \\
\text { vidad }\end{array}$ & $\begin{array}{c}\text { Distancia para } \\
\text { representati- } \\
\text { vidad granos } \\
\text { enteros }\end{array}$ & $\begin{array}{c}\text { Desviación } \\
\text { estándar repre- } \\
\text { sentatividad }\end{array}$ \\
\hline Tonosí & 1 & $0,566 \mathrm{a}^{\dagger}$ & 0,60 & 2 & $0,644 \mathrm{a}^{\dagger}$ & 0,54 \\
Alanje & 2 & $0,589 \mathrm{a}$ & 0,41 & 7 & $0,830 \mathrm{a}$ & 0,54 \\
Divisa & 3 & $0,633 \mathrm{a}$ & 0,42 & 6 & $0,745 \mathrm{a}$ & 0,59 \\
Penonomé & 4 & $0,647 \mathrm{a}$ & 0,50 & 4 & $0,719 \mathrm{a}$ & 0,42 \\
Remedios & 5 & $0,729 \mathrm{a}$ & 0,34 & 10 & $0,997 \mathrm{a}$ & 0,55 \\
Tanara & 6 & $0,823 \mathrm{a}$ & 0,45 & 3 & $0,708 \mathrm{a}$ & 0,40 \\
Calabacito & 7 & $0,894 \mathrm{a}$ & 0,46 & 5 & $0,729 \mathrm{a}$ & 0,58 \\
Soná & 8 & $0,917 \mathrm{a}$ & 0,70 & 8 & $0,869 \mathrm{a}$ & 0,63 \\
Barú & 9 & $0,977 \mathrm{a}$ & 0,55 & 1 & $0,613 \mathrm{a}$ & 0,44 \\
Changuinola & 10 & $1,050 \mathrm{a}$ & 0,65 & 9 & $0,993 \mathrm{a}$ & 0,61 \\
\hline
\end{tabular}

${ }^{\dagger}$ Medias seguidas por la misma letra no difieren significativamente (Duncan $\alpha=0,05$ ).

representatividad de los ambientes de prueba; considerando ambas características (rendimiento de grano + proporción de granos enteros). En este sentido, la capacidad discriminatoria fue similar en Remedios, Tanara, Alanje, seguidas de Penonomé, Soná, Tonosí, Divisa, Barú y Calabacito, siendo estadísticamente inferior Changuinola. En cuanto, a la representatividad fueron similares Calabacito, Tonosí, seguidas por Barú, Soná, Tanara, Alanje, Remedios y Divisa; las menos representativas son Penonomé y Changuinola (Cuadro 8).

En algunas ocasiones los fitomejoradores suelen tener algún tipo de restricción como limitaciones de recursos financieros, baja disponibilidad de semilla, etc. Entonces es importante priorizar y realizar las selecciones en las localidades de prueba más conveniente para las características consideradas en la selección, y no diluir esfuerzos en aquellas que no aportan significativamente al proceso selectivo.

Las distancias estandarizadas indican que Alanje es una localidad excepcional para la selección de germoplasma de arroz, verificamos que a excepción de Alanje, las localidades más apropiadas para seleccionar cultivares con potencial de rendimiento de grano
(Soná y Barú), no fueron las mismas, que permiten seleccionar para obtener mayor proporción de granos enteros en el molino (Tonosí, Barú y Divisa). Por otro lado, mediante el índice de selección (rendimiento+ granos enteros), se identificaron las localidades Tonosí, Alanje, Calabacito, Soná, Barú, como las más convenientes para seleccionar germoplasma de arroz. Las localidades no presentaron diferencias estadísticas en su capacidad discriminatoria para rendimiento de grano; no obstante, hubo diferencias en cuanto a la representatividad, siendo Calabacito y Changuinola la de mayor y menor representatividad, respectivamente. Las diez localidades presentaron similar capacidad discriminatoria y representatividad para la variable granos enteros. Al combinar rendimiento + granos enteros, es posible separar las localidades más discriminatorias (Remedios, Tanara, Alanje) y las más representativas (Calabacito, Tonosí, Barú). Los resultados de este estudio, son importantes para los trabajos de selección que efectúanos en generaciones tempranas (F6, F7 y F8), cuando disponemos de pocas semillas y deseamos evaluar viveros en ambientes que permitan identificar los genotipos superiores. También tiene implicaciones en la utilización de aquellos 
Cuadro 8. Distancias estandarizadas y desviaciones estándar para capacidad discriminatoria y representatividad para ambas características rendimiento de grano de arroz + proporción de granos enteros, en las localidades evaluadas. Instituto de Investigación Agropecuaria de Panamá (IDIAP). 2001-2009.

\begin{tabular}{lcccccc}
\hline Localidades & $\begin{array}{c}\text { Clasificación } \\
\text { capacidad dis- } \\
\text { criminatoria }\end{array}$ & $\begin{array}{c}\text { Distancia para } \\
\text { capacidad dis- } \\
\text { criminatoria } \\
\text { Rend+GE }\end{array}$ & $\begin{array}{c}\text { Desviación } \\
\text { estándar capa- } \\
\text { cidad discrimi- } \\
\text { natoria }\end{array}$ & $\begin{array}{c}\text { Clasificación } \\
\text { representati- } \\
\text { vidad }\end{array}$ & $\begin{array}{c}\text { Distancia para } \\
\text { representatividad } \\
\text { Rendimiento+ } \\
\text { granos enteros }\end{array}$ & $\begin{array}{c}\text { Desviación } \\
\text { estándar repre- } \\
\text { sentatividad }\end{array}$ \\
\hline Remedios & 1 & $0,616 \mathrm{a}^{\dagger}$ & 0,23 & 7 & $0,832 \mathrm{abc}^{\dagger}$ & 0,46 \\
Tanara & 2 & $0,641 \mathrm{a}$ & 0,24 & 5 & $0,727 \mathrm{ab}$ & 0,26 \\
Alanje & 3 & $0,672 \mathrm{a}$ & 0,35 & 6 & $0,737 \mathrm{ab}$ & 0,40 \\
Penonomé & 4 & $0,726 \mathrm{ab}$ & 0,26 & 9 & $0,954 \mathrm{bc}$ & 0,34 \\
Soná & 5 & $0,777 \mathrm{ab}$ & 0,46 & 4 & $0,719 \mathrm{ab}$ & 0,31 \\
Tonosí & 6 & $0,792 \mathrm{ab}$ & 0,56 & 2 & $0,632 \mathrm{a}$ & 0,21 \\
Divisa & 7 & $0,805 \mathrm{ab}$ & 0,33 & 8 & $0,852 \mathrm{abc}$ & 0,41 \\
Barú & 8 & $0,918 \mathrm{ab}$ & 0,35 & 3 & $0,711 \mathrm{ab}$ & 0,46 \\
Calabacito & 9 & $0,926 \mathrm{ab}$ & 0,49 & 1 & $0,630 \mathrm{a}$ & 0,40 \\
Changuinola & 10 & $1,018 \mathrm{c}$ & 0,45 & 10 & $1,100 \mathrm{c}$ & 0,19 \\
\hline
\end{tabular}

${ }^{\dagger}$ Medias seguidas por la misma letra no difieren significativamente (Duncan $\alpha=0,05$ ).

ambientes que son los más representativos de las zonas arroceras cuando deseamos hacer estudios bien específicos sobre la respuesta de nuestro germoplasma a estos ambientes.

El estudio refleja que las zonas productoras de arroz en Panamá, representadas por las diez localidades constituyen ambientes complejos. La estimación de las distancias estandarizadas entre las localidades verdaderas y la ideal puede ser un parámetro importante para determinar la fortaleza de los ambientes para hacer más efectivo el trabajo de selección y/o recomendación de nuevos cultivares. La separación de las localidades respecto a la capacidad discriminatoria y representatividad provee información útil sobre la efectividad o conveniencia de las localidades para el desarrollo y selección de germoplasma y/o recomendar cultivares con adaptación amplia o específica.

\section{AGRADECIMIENTOS}

Al Instituto de Investigación Agropecuaria de Panamá (IDIAP), al Fondo Regional de Tecnología
Agropecuario (FONTAGRO), por el apoyo financiero para hacer el estudio y a los colegas investigadores del IDIAP, por su excelente trabajo en la evaluación del germoplasma durante el periodo que comprende el estudio.

\section{LITERATURA CITADA}

Atlin, GN; Mcrae, KB; Lu, X. 2000. Genotype x region interaction for two-row barley yield in Canada. Crop Science 40:1-6.

Baker, RJ. 1988. Tests for crossover genotype-by-environment interactions. Canadian Journal of Plant Science 68:405-410.

Baxevanos, D; Goulas, C; Rossi, J; Braojos E. 2008. Separation of cotton cultivar testing sites based on representativeness and discriminating ability using GGE Biplot. Agronomy Journal 100:1230-1236.

Blanche, SB. 2005. New methods to assess cotton varietal stability and identify discriminating environments. $\mathrm{Ph} . \mathrm{D}$. Thesis. Louisiana, Louisiana State University, USA. 92 p. 
Blanche, SB; Myers, GO. 2006. Identifying discriminating locations for cultivar selection in Louisiana. Crop Science 46:946-949.

Crossa, J. 1990. Statistical analysis of multilocation trials. Advances in Agronomy 44:55-85.

Cruz, CD; Torres, RA. de; Vencovsky, R. 1989. An alternative approach to the stability analysis proposed by Silva and Barreto. Revista Brasileira de Genética 12:567-580.

Eberhart, SA; Russell, WA. 1966. Stability parameters for comparing varieties. Crop Science 6:36-40.

Finlay, KW; Wilkinson GN. 1963. The analysis of adaptation in a plant breeding programme. Australian Journal of Agricultural Research 14:742-754.

Fox, PN; Rosielle AA. 1982. Reducing the environmental main effects on pattern analysis of plant breeding environments. Euphytica 31:645-656.

Gauch, HG; Zobel, RW. 1997. Identifying megaenvironments and targeting genotypes. Crop Science 37:311-326.

Glaz, B; Kang, MS. 2008. Location contributions determined via GGE biplot analysis of multienvironment sugarcane genotype-performance trials. Crop Science 48:941-950.

Kang, MS. 1993. Simultaneous selection for yield and stability in crop performance trials: Consequences for growers. Agronomy Journal 85:754-757.

Lillemo, M; Ginke LM Van; Trethowan, RM; Hernández, E; Rajaram, S. 2004. Associations among International CIMMYT bread wheat yield testing locations in high rainfall areas and their implications for wheat breeding. Crop Science 44:1163-1169.

Lin, CS. 1982. Grouping genotypes by a cluster method directly related to genotype-environment interaction mean square. Theoretical and Applied Genetics 62:277-280.

Lin, CS; Binns, MR. 1988. A superiority measure of cultivar performance for cultivar $\mathrm{x}$ location data. Canadian Journal of Plant Science 68:193-198.

MIDA (Ministerio de Desarrollo Agropecuario). 2010. Dirección Nacional de Agricultura. Programa Nacional de Granos Básicos. Cultivo de Arroz Mecanizado
Secano y Riego. Informe Final del Año Agrícola 20092010. Panamá. 31 p.

Peterson, CJ; Pfeiffer, WH. 1989. International winter wheat evaluation: Relationships among test sites based on cultivar performance. Crop Science 29:276-282.

Rodríguez-Pérez, JE; Sahagún-Castellanos, J; VillaseñorMir, HE; Molina-Galán, JD; Martínez-Garza, A. 2005. La interacción genotipo $\times$ ambiente en la caracterización de áreas temporaleras de producción de trigo. Agrociencia 39:51-64.

Thomason, WE; Phillips, SB. 2006. Method to evaluate cultivar testing environments and improve cultivar selection protocols. Field Crops Research 99:87-95.

Trethowan, RM; Ginkel, LM Van; Ammar, K; Crossa, J; Payne, TS; Cukadar, B; Rajaram, S; Hernandez, E. 2003. Associations among twenty years of international bread wheat yield evaluation environments. Crop Science 43:1698-1711.

Westcott, B. 1987. A methods of assessing the yield stability of crop genotypes. Journal of Agricultural Science 108:267-274.

Yan, W; Hunt, LA; Sheng, Q; Szlavnics, Z. 2000. Cultivar evaluation and mega-environment investigation based on the GGE Biplot. Crop Science 40:597-605.

Yan, W; Cornelius, PL; Crossa, J; Hunt LA. 2001. Two types of GGE Biplots for analyzing multi-environment trial data. Crop Science 41:656-663.

Yan, W; Rajcan, I. 2002. Biplot evaluation of test sites and trait relations of soybean in Ontario. Crop Science 42:11-20.

Yan,W; Hunt,LA. 2002. Biplot analysis of multi-environment trial data. In KANG, MS. ed. Quantitative genetics, genomics and plant breeding. CAB International. p.289-319.

Yan, W; Kang, MS. 2003. GGE Biplot Analysis: A Graphical tool for breeders, geneticist, and agronomists. CRC Press. Boca Raton, F.L. 271 p.

Yang, RC; Blade, SF; Crossa, J; Stanton, D; Bandara, MS. 2005.Identifying isoyield environments for field pea production. Crop Science 45:106-133.

Zobel, RW; Wright, MJ; Gauch, HG. 1988. Statistical analysis of a yield trial. Agronomy Journal 80:388-393. 